\title{
Interference pattern signatures in fully differential cross sections for single ionization of $\mathrm{H}_{2}$ molecules by fast protons
}

\author{
M F Ciappina ${ }^{1}$ and R D Rivarola ${ }^{2}$ \\ ${ }^{1}$ Max Planck Institute for the Physics of Complex Systems, Nöthnitzer Str 38, D-01187 Dresden, \\ Germany \\ ${ }^{2}$ Instituto de Física Rosario (CONICET-UNR), Av. Pellegrini 250, 2000 Rosario, Argentina \\ E-mail: ciappi@pks.mpg.de
}

Received 22 September 2007, in final form 10 November 2007

Published 19 December 2007

Online at stacks.iop.org/JPhysB/41/015203

\begin{abstract}
Electron interference signatures present in fully differential cross sections for single ionization by $6 \mathrm{MeV}$ protons in $\mathrm{H}_{2}$ molecules are investigated. We employ a molecular version of the continuum-distorted wave-eikonal initial state model, where all the interactions present in the exit channel are considered on an equal footing. Calculations of fully differential cross sections are performed for different electron and projectile kinematical conditions and the range of validity of the theoretical approach is discussed. Furthermore, we explore the presence of interference patterns in differential cross sections for both aligned and randomly oriented targets in asymmetric coplanar geometries.
\end{abstract}

\section{Introduction}

One of the most active topics in molecular physics nowadays is the interaction of charged particles or photons with simple and complex molecules. These processes are of fundamental interest in the subsequent study in many fields of research, e.g. in atmospheric, plasma and biological physics. It is also of interest to obtain information about the molecular structure using different probes; amongst them we can cite photons [1-8], electrons [9-14] or heavy ions [15-21]. The first experimental evidence of the existence of interference patterns in single ionization of molecules by ion impact was given by Stolterfoht et al [15]. In this work, double differential cross sections (DDCS) for single ionization of $\mathrm{H}_{2}$ molecules by fast $\mathrm{Kr}^{34+}$ beams as a function of the momentum of the ionized electron were measured. The theoretical predictions applying distorted wave theories confirmed that the oscillatory behaviour of the experimental data could be explained in terms of coherent electron emission from the proximities of the two atomic centres composing the target.

Fragmentation of molecules by charged particles, i.e. ions and electrons, is also relevant from a more fundamental point of view, since this process configures a nontrivial quantummechanical many-body problem, where Coulomb interactions play an important role. There are several differences between strong laser ionization and processes induced by fast charged particles. First, the time scale where the ionization takes place in the case of ions and electrons is much shorter (of the order of a few fs $\left(10^{-15} \mathrm{~s}\right)$ ) since the projectile produces an electric pulse of a few attoseconds and in this time scale interacts with the atomic electrons and probes simultaneously the correlated electron dynamics in the target, while the residual target remains in some sense frozen, i.e. is not influenced by the projectile. Furthermore, in strong laser fields the ground molecular states are modified (dressed), which yields a considerable amount of additional complications in the theoretical modelling of such processes. Consequently, we can consider charged particles as much cleaner when interacting with molecules than ultrashort laser pulses. We additionally note that the studies with fast ions are even more attractive, due to the absence of exchange (e.g. electron-electron correlation) effects, which appear when we use electrons as probes.

The understanding of atomic and molecular processes has been strongly benefited since the advent of the COLTRIMS (cold target recoil ion momentum spectroscopy) and reaction microscope techniques, which now allow simultaneous detection of the different particles that participate in such processes (for a review see [22]). Thus, the complete kinematics of a huge amount of experiments can be determined by measuring the momenta of all fragments in coincidence. 
An additional advantage is that the target beams (molecules or atoms) produced by the supersonic expansion are intrinsically cold and therefore in their ground state in the case of atoms, and practically in a vibrational and in many cases also rotational ground states for molecules. Fully differential data for electron emission and molecular frame photo-electron angular distributions have been obtained already for photon impact [23]. To deal with single impact molecular ionization by charged particles, i.e. ions or electrons, the experimental scenario is more complicated since more particles have to be detected in the final state in order to obtain kinematically complete data (e.g. in a single molecular ionization we have to detect the ionized electron in coincidence with the recoilresidual molecular ion) on one hand and, on the other hand, the amount of energy transferred by the projectile to the molecular target is not fixed. Furthermore, recent experiments have revealed the important role of autoionization channels on the emission of very low-energy electrons for the simplest molecule $\mathrm{H}_{2}$ [19]. Theoretical approaches to deal with ionization or fragmentation of molecules by ion impact are a formidable task as well, and only a few predictions have been made up to now for simple molecules. For fast ion impact the situation is somewhat more tractable since typically very little momentum is transferred from the projectile to the molecular target during the collision and ionization might become very similar or even equivalent to photoionization. This limit has been extensively discussed for atomic targets starting with the original work of Inokuti [24].

In all theoretical treatments of single ionization of molecular species knowledge of the initial bound and final continuum multielectronic states is required. Due to the complexity of these problems and, in order to avoid difficulties with dealing of multielectronic targets, the most widely used approach is to reduce the problem to a one-active electron description. This approximation has been successfully applied in different molecular and atomic processes. Furthermore, there exist powerful computational codes that allow us to obtain an accurate ground molecular state as a combination of atomic orbitals. On the other hand, the continuum for the ionized electron is more complicated to treat, since the interaction between the electron and the residual-molecularion presents a Coulomb-based multi-body problem. In this sense, since the aim of our study is to capture the structural information of the molecular target, we use simple models for the ionized electron and we concentrate our analysis on one of the simple molecules $\left(\mathrm{H}_{2}\right)$. In a first approach it is possible to consider the ionized electron in an effective field produced by the residual ionic core, screened by the other passive electrons. This last approach, known as the distorted-waveBorn approximation (DWBA), has been successfully used to model double differential cross sections in di- and polyatomic single ionization processes (see [18] and references therein). In an even simpler approach it is possible to consider the ionized electron as free, i.e using a plane-wave approximation (PW). PW has been used extensively in strong field induced processes in molecules (see, e.g., [3] and references therein).

In collisions of simple molecules with heavy ions it is possible to apply more elaborate schemes to describe the single ionization process. In this sense it has been shown that the continuum-distorted wave-eikonal initial state (CDWEIS) of Crothers and McCann [25] can be extended to the treatment of collisions of $\mathrm{H}_{2}$ with heavy particles (see, e.g., [17] and references therein). Furthermore, within the CDWEIS scheme, it is possible to incorporate the interaction between the heavy nuclei, also known as $\mathrm{N}-\mathrm{N}$ interaction, in a semiclassical way [26]. It has been shown that this approach is able to reproduce with reasonable agreement fully differential cross sections (FDCS) for single ionization of helium by heavy ions in a broad range of projectile velocities (see, e.g., [27-30]).

Another point to mention is the alignment dependence of the laser- and atomic-induced processes. In all of the studied cases up to now only randomly oriented ensembles of molecules were used (see [21] and references therein). On the other hand, using a pump-probe technique, it would be perfectly feasible to perform experiments with strongly aligned molecules, as in the case of strong laser-induced processes $[31,32]$. This technique has reached high maturity and nowadays there is a large amount of experiments associated with it. Even when the case of pump-probe experiments using fast ions as probe is experimentally challenging, we consider it appropriate to predict the features that will emerge from these incoming outcomes.

In the following section, we briefly describe our theoretical framework to deal with ion- $\mathrm{H}_{2}$ collisions. In section 3, we report results corresponding to physical conditions which are perfectly suitable in future experiments. A discussion about the changes produced by the presence of multiple atomic centres in the main structures of the FDCS in single ionization of molecules by proton impact, i.e. the binary and recoil peaks, is considered. Finally in section 4 we write our conclusions, joint with some perspectives. Atomic units are used throughout unless otherwise stated.

\section{Theory}

Let us consider single ionization of $\mathrm{H}_{2}$ molecules by an impact of a bare nucleus of charge $Z_{P}$ and velocity $\mathbf{v}$. The geometry of the collision system is shown in figure 1. The incoming projectile beam is directed along the $z$-axis and we define the $x-z$ as the scattering plane. The orientation of the momentum $\mathbf{k}$ of the ionized electron is represented in usual spherical coordinates by the angles $\theta_{e}$ and $\phi_{e}$. Finally, the angle $\alpha$ defines the orientation of the internuclear axis with respect to the $z$-axis (see the following section).

We will analyse the FDCSs as a function of the final electron momentum $\mathbf{k}$ and the transverse component $\boldsymbol{\eta}$ of the momentum transfer $\mathbf{q}=\mathbf{K}_{\mathrm{i}}-\mathbf{K}_{\mathrm{f}}$, where $\mathbf{K}_{\mathrm{i}}\left(\mathbf{K}_{\mathrm{f}}\right)$ is the initial (final) momentum of the incoming projectile. In our context $\boldsymbol{\eta} \cdot \widehat{\mathbf{v}}=0, \widehat{\mathbf{v}}$ being the direction of the vector $\mathbf{v}$. The FDCS is connected to the transition amplitude $T_{\mathrm{fi}}^{(-)}(\boldsymbol{\eta}, \mathbf{R})$ by energy conservation as

$\sigma^{5}(\mathbf{k}, \mathbf{q}, \mathbf{R})=\frac{\mathrm{d}^{5} \sigma}{\mathrm{d} \mathbf{k} \mathrm{d} \boldsymbol{\eta}}=\frac{(2 \pi)^{4}}{v^{2}}\left|T_{\mathrm{fi}}^{(-)}(\boldsymbol{\eta}, \mathbf{R})\right|^{2} \delta\left(E_{\mathrm{f}}-E_{\mathrm{i}}\right)$,

where $\mathbf{R}$ is the vector that identifies the relative position of the nuclei in the molecule. In expression (1), the interaction 


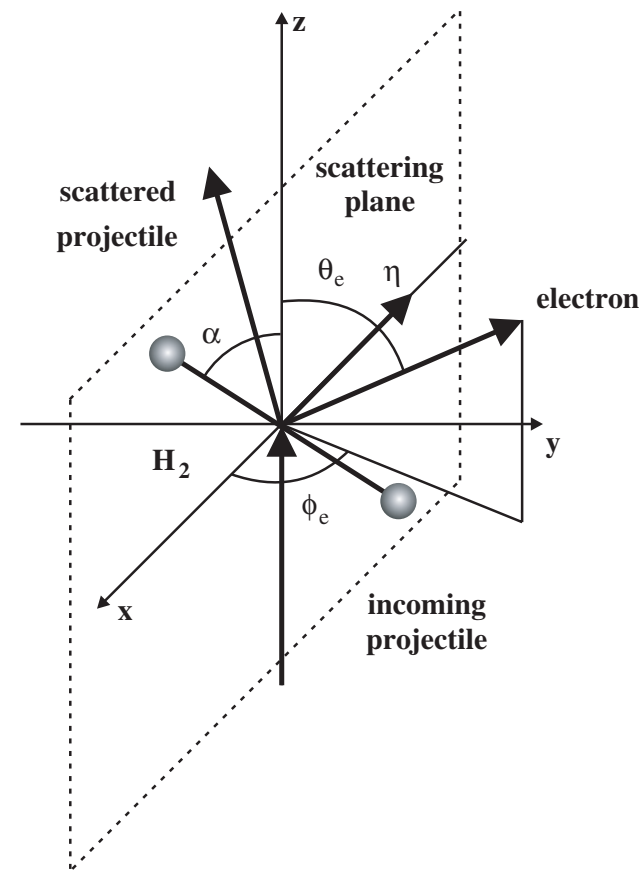

Figure 1. Coordinate system and angles used in the present work. The $z$-axis is parallel to the direction of the incoming projectile and the $x-z$ plane defines the scattering plane. The internuclear axis of the $\mathrm{H}_{2}$ molecule subtends an angle $\alpha$ with respect to the $z$-axis.

between the projectile and each one of the nuclei of the molecule must be included. It was not necessary in previous calculation of cross sections differential in the energy and solid angle subtended by the ejected electron [33]. We will not go into detail about the CDW-EIS formulation extended to $\mathrm{H}_{2}$ molecules (CDW-EIS MO) since the full approach can be found in, e.g. [18]. We only summarize the main points that will be important in the subsequent analysis.

According to the separation in centres of the CDWEIS MO the transition amplitude $T_{\mathrm{fi}}^{(-)}(\boldsymbol{\eta}, \mathbf{R})$ is a sum of two terms which correspond to an emission out of effective atomic wavefunctions centred on nucleus 1 and 2, respectively. Consequently, within the CDW-EIS MO approach, $T_{\mathrm{fi}}^{(-)}(\boldsymbol{\eta}, \mathbf{R})$ can be written as

$$
\left|T_{\mathrm{fi}}^{(-)}(\boldsymbol{\eta}, \mathbf{R})\right|^{2}=2\{1+\cos [(\mathbf{k}-\mathbf{q}) \cdot \mathbf{R}]\}\left|T_{\mathrm{fi}}^{\mathrm{eff},(-)}(\boldsymbol{\eta})\right|^{2} .
$$

Finally, $T_{\mathrm{fi}}^{\mathrm{eff},(-)}(\boldsymbol{\eta})$ is the CDW-EIS transition amplitude corresponding to effective atomic centres located at the position of each molecular centre. We note that in single ionization of $\mathrm{H}_{2}$ by electron impact a similar formula can be obtained [9]. The case of heavy-ion impact ionization is easier to treat, since the projectile can be considered classically and we do not have to deal with exchange effects in the final electronic state. Furthermore, within $T_{\mathrm{fi}}^{\text {eff, }(-)}(\boldsymbol{\eta})$ the interaction between the heavy nuclei, i.e. the Coulomb interaction among the projectile and each atomic centre, can be incorporated exactly in a semiclassical way [26].

In a similar form that was performed in, e.g. [17], for the case of doubly differential cross sections, we can factorize the FDCSs as follows:

$$
\sigma^{5}(\mathbf{k}, \mathbf{q}, \mathbf{R})=\sigma_{\mathrm{d}}^{5}(\mathbf{k}, \mathbf{q})+\sigma_{\mathrm{i}}^{5}(\mathbf{k}, \mathbf{q}, \mathbf{R}) .
$$

From this last equation we can extract the contributions of the direct term $\left(\sigma_{\mathrm{d}}^{5}(\mathbf{k}, \mathbf{q})\right)$ and the interference one $\left(\sigma_{\mathrm{i}}^{5}(\mathbf{k}, \mathbf{q}, \mathbf{R})\right)$, respectively. We note that the last formulation corresponds to the case of aligned molecules, i.e. fixing the nuclei in a spatial orientation.

For the case of randomly oriented molecules an average over all the molecular orientations should be performed in (1). Consequently, we obtain the transition amplitude $T_{\mathrm{fi}}^{\mathrm{r},(-)}(\boldsymbol{\eta}, \mathbf{R})$ as

$\left|T_{\mathrm{fi}}^{\mathrm{r},(-)}(\boldsymbol{\eta}, \mathbf{R})\right|^{2}=8 \pi\left\{1+\frac{\sin (|\mathbf{k}-\mathbf{q}| R)}{|\mathbf{k}-\mathbf{q}| R}\right\}\left|T_{\mathrm{fi}}^{\mathrm{eff},(-)}(\boldsymbol{\eta})\right|^{2}$,

being $T_{\mathrm{fi}}^{\mathrm{eff},(-)}(\boldsymbol{\eta})$ the same transition amplitude as in the case of oriented molecules.

\subsection{Ratios}

The usual procedure to observe the interference behaviour in differential cross sections is to relate molecular data with their respective atomic counterparts [15, 17, 18]. According to the formulation explained above, interference terms in single ionization of $\mathrm{H}_{2}$ targets will appear when the molecular FDCSs (1) are divided by twice FDCSs for atomic $\mathrm{H}$ targets. If there are no effects due to the molecular structure, we can expect that the ratio will give a value close to 1 (it might differ from this value due to the different binding energies of $\mathrm{H}_{2}$ and $\mathrm{H}$, the effective charges $Z_{\text {eff }}$ used to model the final molecular continuum state and the normalization of the bound-state wavefunctions).

Besides the small differences presented between the ratio among the molecular and their atomic counterparts, we can isolate interference factors that allow a more detailed study. In that sense we can define for the case of oriented molecules a $\operatorname{term} F_{\mathrm{o}}^{\alpha,\left(\theta_{e}, \phi_{e}\right)}(\mathbf{k}, \mathbf{q}, \mathbf{R})$,

$$
F_{\mathrm{o}}^{\alpha,\left(\theta_{e}, \phi_{e}\right)}(\mathbf{k}, \mathbf{q}, \mathbf{R})=\{1+\cos [(\mathbf{k}-\mathbf{q}) \cdot \mathbf{R}]\},
$$

where we have explicitly indicated the dependence of $F_{\mathrm{o}}^{\alpha,\left(\theta_{e}, \phi_{e}\right)}(\mathbf{k}, \mathbf{q}, \mathbf{R})$ on the molecular orientation angle $(\alpha)$ and the electron angles $\theta_{e}, \phi_{e}$ (see figure 1 for details). On the other hand, for randomly oriented targets, analysing (4) we can extract $F_{\mathrm{r}}(\mathbf{k}, \mathbf{q}, \mathbf{R})$,

$$
F_{\mathrm{r}}(\mathbf{k}, \mathbf{q}, \mathbf{R})=\left\{1+\frac{\sin (|\mathbf{k}-\mathbf{q}| R)}{|\mathbf{k}-\mathbf{q}| R}\right\} .
$$

In the following section we will perform calculations using (1) for the case of single ionization of $\mathrm{H}_{2}$ by proton impact. However, the present formalism is also valid to evaluate FDCS for single ionization produced by multiply charged projectiles. Moreover, it is well known that the CDW-EIS represents a suitable theory to model these collision processes (see, e.g., [34]). Furthermore, a detailed study of the properties of the terms (5) and (6) for different values of the electron $\mathbf{k}$ and transfer $\mathbf{q}$ momenta is accomplished. 


\section{Results and discussion}

We have applied the CDW-EIS MO to single ionization of $\mathrm{H}_{2}$ by $6 \mathrm{MeV}(v=15.5 \mathrm{au})$ protons which follow the experimental set-up of [19]. We have chosen the electron and projectile parameters in order to allow comparisons between our predictions and possible future experiments (see the discussion below). The pure ionization of the $\mathrm{H}_{2}$ molecule is modelled using an equilibrium distance $R=$ $1.4 \mathrm{au}$ and the initial electronic state in each centre is given by a hydrogenic function with a variational charge $Z=1.19$ and the corresponding normalization factor $N_{\mathrm{i}}(R)=0.5459$. For the final electronic state, continuum wavefunctions centred on each target nucleus are used with an effective charge $Z_{\text {eff }}=\sqrt{-2 \epsilon_{\mathrm{i}}}$, being $\epsilon_{\mathrm{i}}=-0.566$ au the initial binding energy [19].

Our next step will be to disentangle the details contained in the interference term for the case of oriented molecules. To this end, we note that the cosine term in (5) gives the interferences contribution. Considering that the molecular axis lies in the collision plane, we can write the argument of the cosine function of (5) in spherical coordinates

$$
\begin{aligned}
& (\mathbf{k}-\mathbf{q}) \cdot \mathbf{R} \\
& =\left(k \sin \theta_{e} \cos \phi_{e}+q, k \sin \theta_{e} \sin \phi_{e}, k \cos \theta_{e}+q_{\min }\right) \\
& \quad \cdot(R \sin \alpha, 0, R \cos \alpha),
\end{aligned}
$$

where $q_{\min }=\Delta \epsilon / v$, with $\Delta \epsilon=\epsilon_{\mathrm{f}}-\epsilon_{\mathrm{i}}$ and $\epsilon_{\mathrm{f}}=k^{2} / 2$ the final electronic energy. Let us note that the direction of the momentum transfer $\mathbf{q}$ lies almost in the negative $x$ axis [35]. In the following, we specialize our analysis in the most important structures that appear in the in-plane FDCS for single ionization: the binary and recoil peaks. The former appear as a consequence of a binary collision between the projectile and the active electron. On the other hand, the latter is a consequence of a two-step process: first the projectile collides with the electron and after that electron interacts with the nuclear target. Both structures are well reproduced by the CDW-EIS theory in the range of parameters we analyse [20].

\subsection{Binary peak}

In the case of ionization due to ion impact at high velocities, the electron angles of the binary peak can be considered as $\theta_{e} \approx \pi / 2$ and $\phi_{e}=\pi$ [35]. Consequently, expanding (7) we have: $(\mathbf{k}-\mathbf{q}) \cdot \mathbf{R}=-k R \sin \alpha+q_{\min } R \cos \alpha+q R \sin \alpha$.

To proceed it is necessary to freeze our $\mathrm{H}_{2}$ molecule in space. At present, this procedure is experimentally difficult for the case of small molecules. However, recently pumpprobe experiments with $\mathrm{N}_{2}$ and $\mathrm{CO}_{2}$ molecules within the framework of strong laser physics were reported [31, 32]. We expect that in the near future, it would be perfectly feasible to perform experiments using a pump pulse to produce a strongly oriented ensemble of $\mathrm{H}_{2}$ molecules and after that to collide such molecules with a collimated beam of heavy ions. Theoretically, we will consider two cases: (i) the molecule is aligned parallel to the beam direction, i.e. $\alpha=0$, since the projectile velocity has only a component in the $z$-axis and (ii) the molecule is aligned perpendicular to the beam direction, i.e. $\alpha=\pi / 2$. Even when it could be too restrictive to analyse only two cases, our predictions can be easily extended to other orientations.

By considering the parallel case $(\alpha=0)$ we can write (5) as

$$
F_{\mathrm{o}}^{0,(\pi / 2, \pi)}(\mathbf{k}, \mathbf{q}, \mathbf{R})=\left[1+\cos \left(q_{\min } R\right)\right] .
$$

The values of $F_{\mathrm{o}}^{0,(\pi / 2, \pi)}(\mathbf{k}, \mathbf{q}, \mathbf{R})$ for two typical values of $q$ ( $q=0.5$ au and $q=0.8 \mathrm{au})$ using $E_{e}=10 \mathrm{eV}(k=0.86 \mathrm{au})$ and $E_{e}=30 \mathrm{eV}(k=1.48 \mathrm{au})$ are approximately 2 for all cases. In figure 2 we plot the corresponding FDCS, disentangling the contribution of the direct $\left(\sigma_{\mathrm{d}}^{5}(\mathbf{k}, \mathbf{q})\right)$ term, i.e the term that represents an incoherent contribution of two effective $\mathrm{H}$ atoms. We can see that in all cases the binary peak is enhanced around a factor 2 due to a constructive interference between the two atomic centres.

For the perpendicular case, i.e. $(\alpha=\pi / 2)$, the interference factor (5) reads

$$
F_{\mathrm{o}}^{\pi / 2,(\pi / 2, \pi)}(\mathbf{k}, \mathbf{q}, \mathbf{R})=[1+\cos (R(q-k))] .
$$

For the parameter set analysed in the parallel alignment we have $F_{0}^{\pi / 2,(\pi / 2, \pi)}(\mathbf{k}, \mathbf{q}, \mathbf{R})=1.87\left(E_{e}=10 \mathrm{eV}\right.$ and $q=0.5 \mathrm{au}), F_{\mathrm{o}}^{\pi / 2,(\pi / 2, \pi)}(\mathbf{k}, \mathbf{q}, \mathbf{R})=1.20\left(E_{e}=30 \mathrm{eV}\right.$ and $q=0.5 \mathrm{au}), F_{\mathrm{o}}^{\pi / 2,(\pi / 2, \pi)}(\mathbf{k}, \mathbf{q}, \mathbf{R}) \approx 2\left(E_{e}=10 \mathrm{eV}\right.$ and $q=$ $0.8 \mathrm{au})$ and $F_{\mathrm{o}}^{\pi / 2,(\pi / 2, \pi)}(\mathbf{k}, \mathbf{q}, \mathbf{R})=1.58\left(E_{e}=30 \mathrm{eV}\right.$ and $q=$ $0.8 \mathrm{au}$ ), respectively. In figure 3 we show our predictions for this case. It is possible to observe that the binary peak is also enhanced due to a constructive interference contribution from the two atomic centres, being the increasing not so pronounced as in the case of parallel alignment.

\subsection{Recoil peak}

The analysis of the recoil peak can be performed in a similar way. The electron angles for this structure are $\theta_{e} \approx \pi / 2$ and $\phi_{e}=0$. Consequently (7) reads now, $(\mathbf{k}-\mathbf{q}) \cdot \mathbf{R}=$ $k R \sin \alpha-q R \sin \alpha+q_{\min } R \cos \alpha$ and, for parallel alignment $(\alpha=0)$, we have

$$
F_{\mathrm{o}}^{0,(\pi / 2,0)}(\mathbf{k}, \mathbf{q}, \mathbf{R})=\left[1+\cos \left(q_{\min } R\right)\right] .
$$

As happens for the binary peak region, we can deduce that also the recoil peak will be enhanced by the same magnitude, i.e. $F_{\mathrm{o}}^{0,(\pi / 2,0)}(\mathbf{k}, \mathbf{q}, \mathbf{R}) \approx 2$ for all parameters, due to constructive interferences. In figure 2 it is possible to observe clearly this behaviour.

Let us analyse the recoil peak behaviour for perpendicular alignment $(\alpha=\pi / 2)$. Here, $F_{\mathrm{o}}^{\alpha,\left(\theta_{e}, \phi_{e}\right)}(\mathbf{k}, \mathbf{q}, \mathbf{R})$ is

$$
F_{\mathrm{o}}^{\pi / 2,(\pi / 2,0)}(\mathbf{k}, \mathbf{q}, \mathbf{R})=[1+\cos (R(k+q))] .
$$

Replacing the electron and projectile parameters we obtain: $F_{\mathrm{o}}^{\pi / 2,(\pi / 2,0)}(\mathbf{k}, \mathbf{q}, \mathbf{R})=0.67\left(E_{e}=10 \mathrm{eV}\right.$ and $\left.q=0.5 \mathrm{au}\right)$, $F_{\mathrm{o}}^{\pi / 2,(\pi / 2,0)}(\mathbf{k}, \mathbf{q}, \mathbf{R})=0.07\left(E_{e}=30 \mathrm{eV}\right.$ and $\left.q=0.5 \mathrm{au}\right)$, $F_{\mathrm{o}}^{\pi / 2,(\pi / 2,0)}(\mathbf{k}, \mathbf{q}, \mathbf{R})=0.32\left(E_{e}=10 \mathrm{eV}\right.$ and $\left.q=0.8 \mathrm{au}\right)$ and $F_{\mathrm{o}}^{\pi / 2,(\pi / 2,0)}(\mathbf{k}, \mathbf{q}, \mathbf{R})=1.27 \times 10^{-3}\left(E_{e}=30 \mathrm{eV}\right.$ and $q=$ $0.8 \mathrm{au})$. We can extract from these factors that the recoil peak is strongly suppressed, being the most pronounced situation when the argument of the cosine function in (11) is near from $\pi$. In figure 3 it is possible to recognize such suppression. We can attribute this behaviour to a destructive interference of the 

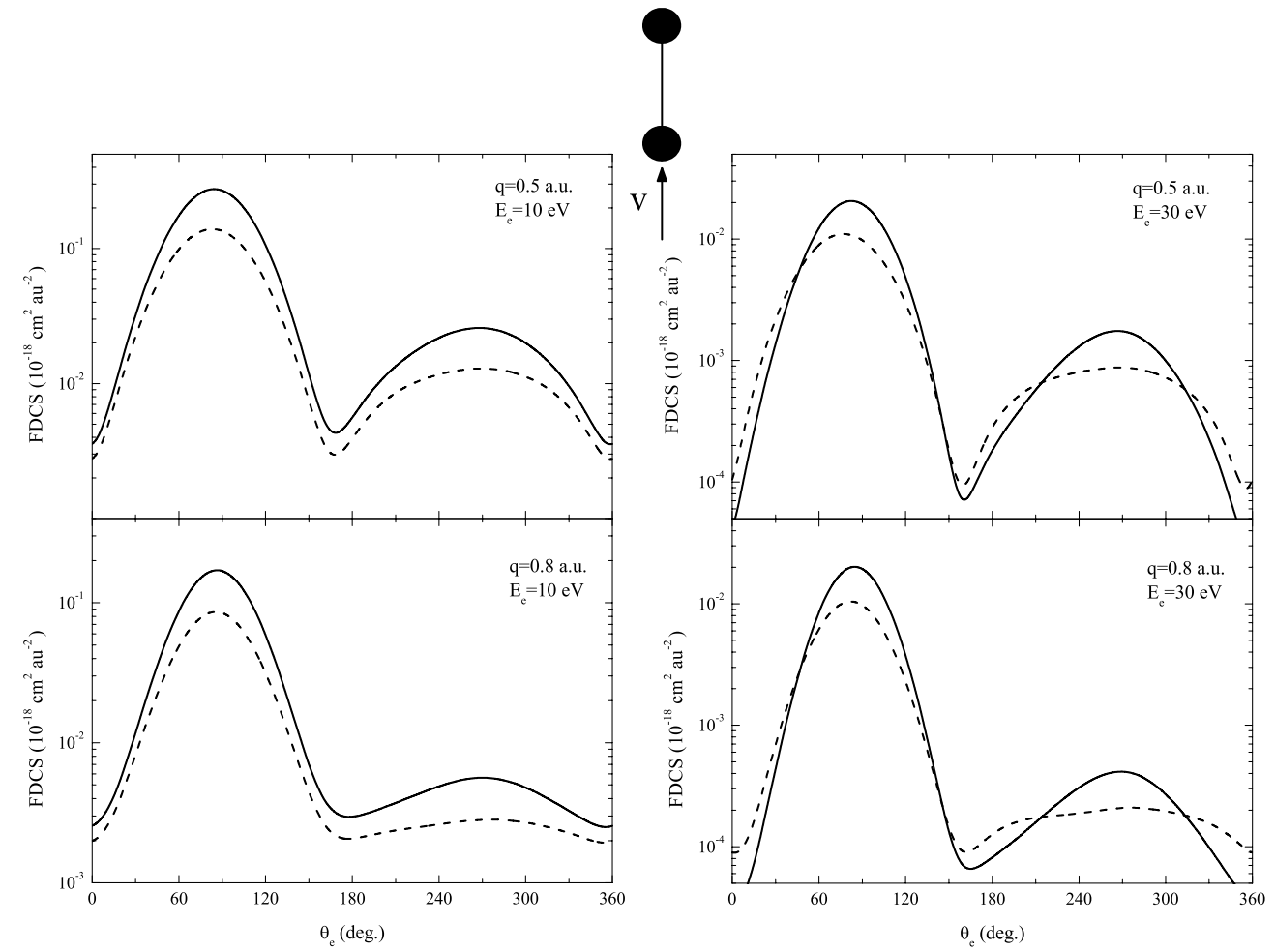

Figure 2. FDCS for single ionization of $\mathrm{H}_{2}$ by $6 \mathrm{MeV}$ protons. The $\mathrm{H}_{2}$ molecule is aligned parallel with respect to the beam direction $(\alpha=0)$. Solid line: total contribution $\left(\sigma^{5}\right)$, dashed line: direct contribution $\left(\sigma_{\mathrm{d}}^{5}\right)$ (see the text).
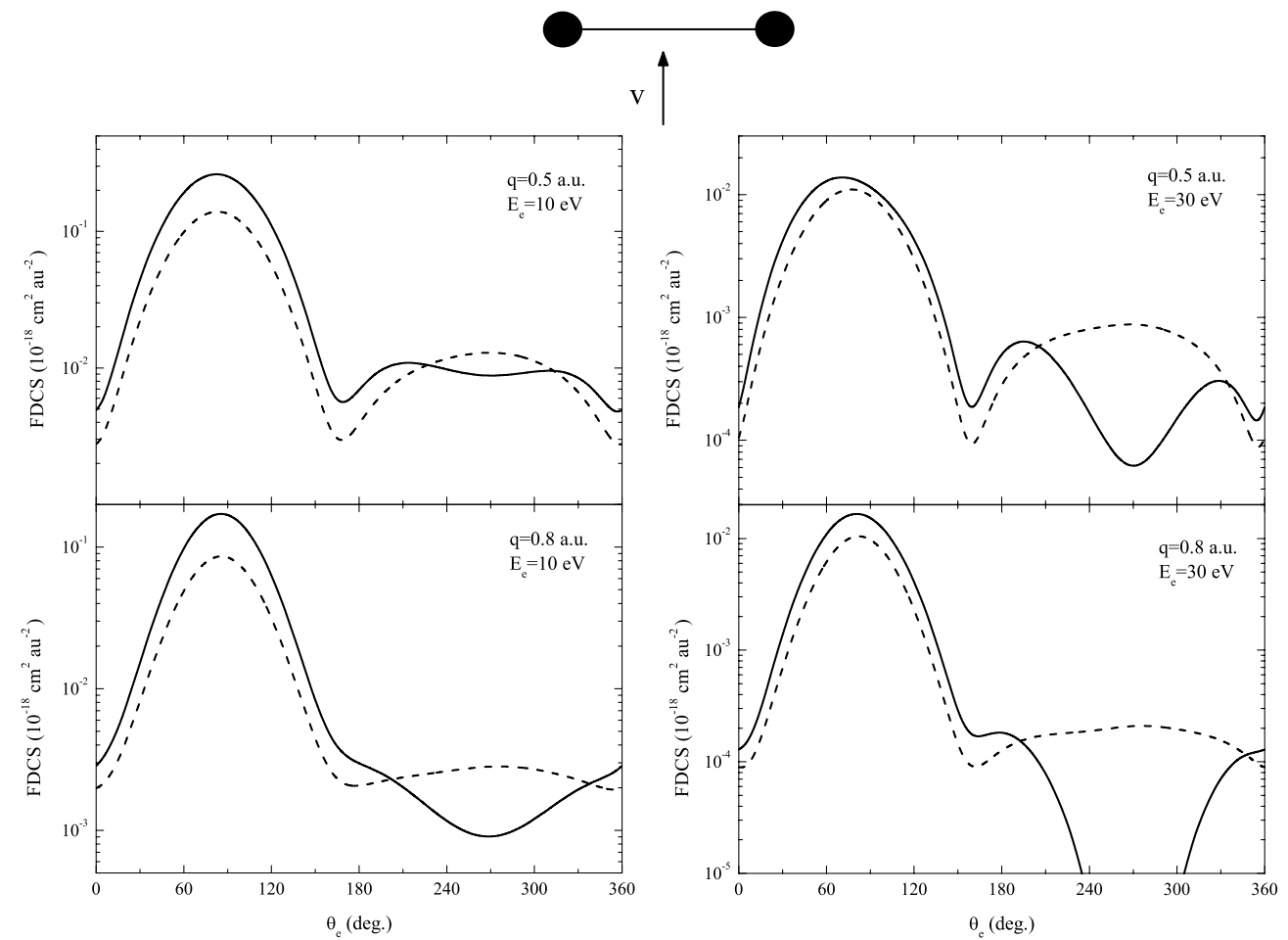

Figure 3. Idem figure 2 for the case of the $\mathrm{H}_{2}$ molecule aligned perpendicular with respect to the beam direction $(\alpha=\pi / 2)$. Solid line: total contribution $\left(\sigma^{5}\right)$, dashed line: direct contribution $\left(\sigma_{\mathrm{d}}^{5}\right)$.

electron emission from the two effective atomic centres, since for this case they are added up out of phase. The existence of destructive interference patterns in the recoil region was also previously discussed in $(\mathrm{e}, 2 \mathrm{e})$ processes for hydrogen molecules $[9,14]$.
The next step is to analyse the randomly oriented case and to study the possibility of predicting interference effects in actual experiments. To this end, in figure 4 we plot FDCS for the case of an ensemble of randomly oriented $\mathrm{H}_{2}$ molecules and for the set of parameters used in figures 2 and 3. From 

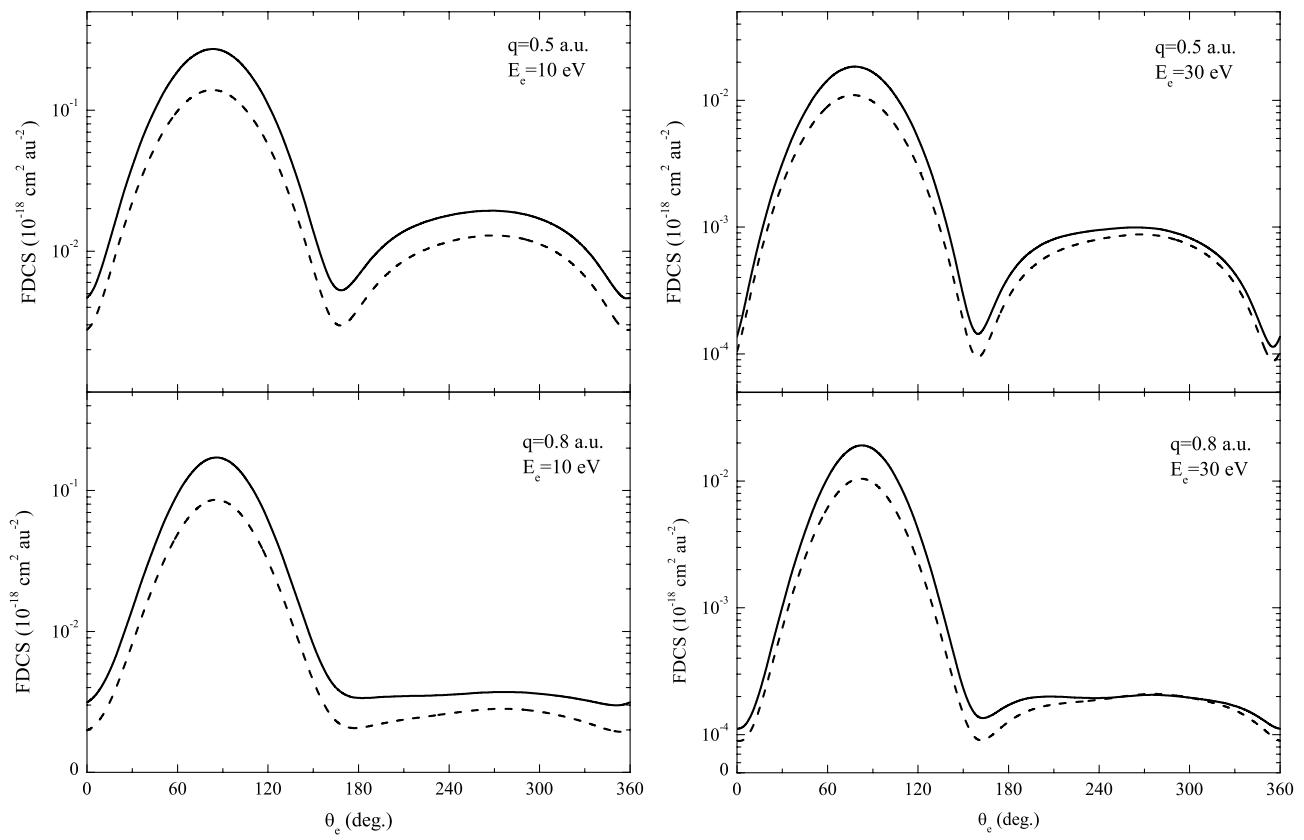

Figure 4. FDCS for single ionization of $\mathrm{H}_{2}$ by $6 \mathrm{MeV}$ protons. The $\mathrm{H}_{2}$ molecule is randomly oriented. Solid line: total contribution $\left(\sigma^{5}\right)$, dashed line: direct contribution $\left(\sigma_{\mathrm{d}}^{5}\right)$.

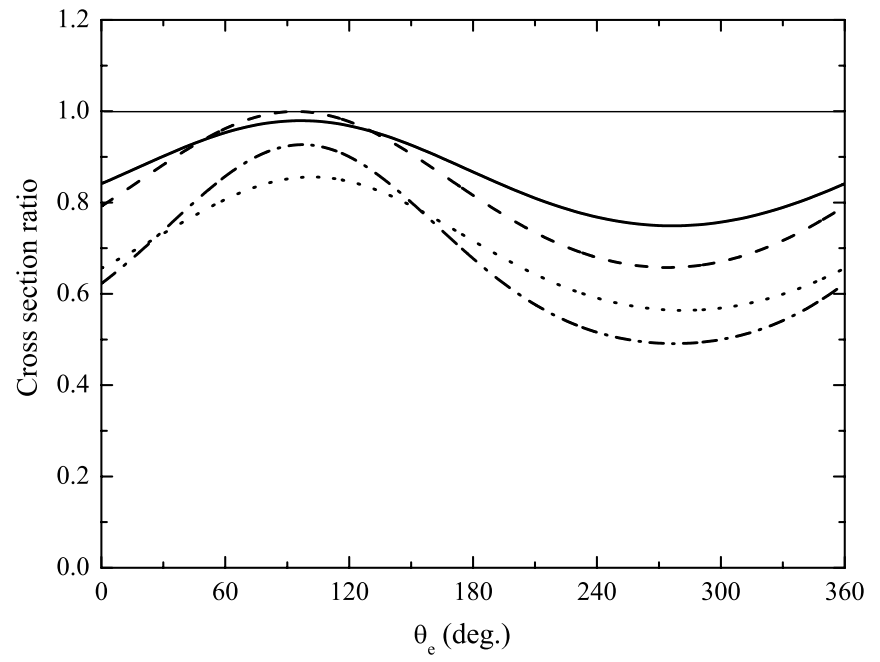

Figure 5. Cross section ratios (see the text for details) for different projectile and electron energy parameters. Solid line: $q=0.5$ au, $E_{e}=10 \mathrm{eV}$, dotted line: $q=0.5 \mathrm{au}, E_{e}=30 \mathrm{eV}$, dashed line: $q=0.8 \mathrm{au}, E_{e}=10 \mathrm{eV}$ and dashed-dotted line: $q=0.8 \mathrm{au}$, $E_{e}=30 \mathrm{eV}$.

the figure we can observe that also for this case there exists an important contribution of the interference term, being more pronounced for the case of lower electron energy, i.e. $E_{e}=10$ $\mathrm{eV}$. We note that the shape of the FDCS for the molecular case is almost similar to that one that appears in atoms with the only difference in magnitude due to the interference contribution.

Finally, in a similar way as was done in Stolterfoht et al [15], the cross section ratios between the $\mathrm{H}_{2}$ FDCS and the atomic counterparts for the case of a randomly oriented ensemble of molecules are considered in figure 5. We have used the same set of parameters for the cases studied above. We can see an oscillatory behaviour that is a fingerprint of the molecular structure. We note that the FDCS are given here as a function of the electron angle $\theta_{e}$, being different than those analysed in, e.g. [15], where the interference structures appeared as a function of the electron energy. To compare our results with experimental ones, and considering that FDCSs for $\mathrm{H}$ atoms are not available nowadays, we could evaluate the experimental-to-theoretical cross section ratio, i.e. to calculate the ratio between the experimental $\mathrm{H}_{2}$ FDCS and the theoretical FDCS for $\mathrm{H}$ atoms, in order to confirm our predictions. From figure 5 we can extract that the most favourable case to observe interferences would be when $E_{e}=30 \mathrm{eV}$ and $q=0.8 \mathrm{au}$, but the other set of parameter studies presents an oscillatory behaviour as well.

\section{Conclusions and perspectives}

We have carried out calculations of FCDSs for single ionization of $\mathrm{H}_{2}$ molecules by fast proton impact. Performing a detailed study of the structures present in the molecular FDCS we were able to disentangle the contribution of the interference term. We predict enhancement of the binary and recoil peaks due to the coherent electron emission from the atomic centres. Moreover, the suppression of the recoil peak can be produced as a consequence of destructive interference of the ionization transition amplitudes coming separately from the proximities of the atomic centres. Furthermore, analysing the ratios between molecular FDCSs and those that result from atomic counterparts we were able to observe oscillations, now as a function of the polar electron angle, that are signatures of molecular interferences.

Even when experimental data for the processes studied here are still unavailable, our predictions and proposals are perfectly feasible with the actual technology. Furthermore, the study of single ionization of aligned molecules shows other 
scenarios to observe interference patterns and presents another tool to characterize the structure and symmetry of molecules.

\section{Acknowledgments}

The authors acknowledge Dr Omar Fojón for fruitful discussions during the completion of the present work. MFC acknowledges the visitor program of the MPIPKS for financial support.

\section{References}

[1] Fojón O A, Fernández J, Palacios A, Rivarola R D and Martín F 2004 J. Phys. B: At. Mol. Opt. Phys. 373035

[2] Rolles D et al 2005 Nature 437711

[3] Jaroń-Becker A, Becker A and Faisal F H M 2004 Phys. Rev. A 69023410

[4] Fojón O A, Palacios A, Fernández J, Rivarola R D and Martín F 2006 Phys. Lett. A 350371

[5] Della Picca R, Fainstein P D, Martiarena M L and Dubois A 2006 J. Phys. B: At. Mol. Opt. Phys. 39473

[6] Della Picca R, Fainstein P D, Martiarena M L and Dubois A 2007 Phys. Rev. A 75032710

[7] Martín F et al 2007 Science 315629

[8] Fernández J, Fojón O, Palacios A and Martín F 2007 Phys. Rev. Lett. 98043005

[9] Stia C R, Fojón O A, Weck P F, Hanssen J and Rivarola R D 2003 J. Phys. B: At. Mol. Opt. Phys. 36 L257

[10] Kamalou O, Chesnel J-Y, Martina D, Hanssen J, Stia C R, Fojón O A, Rivarola R D and Frémont F 2005 Phys. Rev. A 71010702

[11] Gao J F, Madison D H and Peacher J L 2005 Phys. Rev. A 72020701

[12] Gao J F, Madison D H and Peacher J L 2005 Phys. Rev. A 72032721

[13] Gao J F, Madison D H and Peacher J L 2006 J. Phys. B: At. Mol. Opt. Phys. 391275
[14] Milne-Brownlie D S, Foster M, Gao J F, Lohmann B and Madison D H 2006 Phys. Rev. Lett. 96233201

[15] Stolterfoht N et al 2001 Phys. Rev. Lett. 87023201

[16] Laurent G, Fainstein P D, Galassi M E, Rivarola R D, Adoui L and Cassimi A 2002 J. Phys. B: At. Mol. Opt. Phys. 35 L495

[17] Galassi M E, Rivarola R D, Fainstein P D and Stolterfoht N 2002 Phys. Rev. A 66052705

[18] Galassi M E, Rivarola R D and Fainstein P D 2004 Phys. Rev. A 70032721

[19] Dimopoulou C et al 2004 Phys. Rev. Lett. 93123203

[20] Dimopoulou C et al 2005 J. Phys. B: At. Mol. Opt. Phys. 38593

[21] Dimopoulou C et al 2005 J. Phys. B: At. Mol. Opt. Phys. 383173

[22] Ullrich J et al 2003 Rep. Prog. Phys. 661463

[23] Martín F 1999 J. Phys. B: At. Mol. Opt. Phys. 32 R197

[24] Inokuti M 1971 Rev. Mod. Phys. 43297

[25] Crothers D S F and McCann J F 1983 J. Phys. B: At. Mol. Phys. 163229

[26] McCarroll R and Salin A 1968 J. Phys. B: At. Mol. Phys. 1163

[27] Ciappina M F and Cravero W R 2006 J. Phys. B: At. Mol. Opt. Phys. 391091

[28] Ciappina M F and Cravero W R 2006 J. Phys. B: At. Mol. Opt. Phys. 392183

[29] Ciappina M F, Cravero W R and Schulz M 2007 J. Phys. B. At. Mol. Opt. Phys. 402577

[30] Fainstein P D and Gulyás L 2005 J. Phys. B: At. Mol. Opt. Phys. 38317

[31] Itatani J et al 2004 Nature (London) 432867

[32] Kanai T, Minemoto S and Sakai H 2005 Nature (London) 435470

[33] Fainstein P D, Ponce V H and Rivarola R D 1991 J. Phys. B: At. Mol. Opt. Phys. 243091

[34] Stolterfoht N, DuBois R D and Rivarola R D 1997 Electron Emission in Heavy Ion-Atom Collisions (Berlin: Springer)

[35] Madison D H, Schulz M, Jones S, Foster M, Moshammer R and Ullrich J 2002 J. Phys. B: At. Mol. Opt. Phys. 353297 